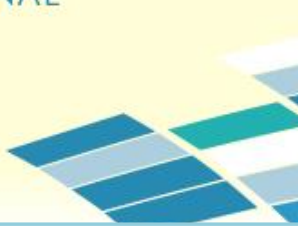

Francisco J. Racero Montes, Manuel Jesús Ramos Corpas \& Manuel Castillo García. Validación de un instrumento de práctica docente no presencial en Formación Profesional

\title{
Validación de un instrumento de práctica docente no presencial en Formación Profesional
}

\section{Validation of the no presential teaching evaluation instrument in Vocational Training}

Francisco J. Racero Montes. Universidad Pablo de Olavide (Sevilla-España). firacmon@upo.es

Manuel Jesús Ramos Corpas. Universidad Pablo de Olavide (Sevilla-España). miramcor@upo.es

Manuel Castillo García. Universidad Hispalense de Sevilla (España). mcastillo23@us.es

\section{RESUMEN.}

La pandemia de COVID-19 está amenazando gravemente la salud de la humanidad provocando consecuencias de toda índole. Un sector bastante afectado, es el educativo. El aprendizaje del alumnado se ha visto reducido en el último cuatrimestre del curso escolar 2019-20, y probablemente los métodos de enseñanza que han tenido que forzarse en este tiempo tengan que repetirse para el curso próximo, si la ciencia no encuentra un remedio rápido para combatir el virus. En este contexto las administraciones públicas en el marco de sus competencias constitucionales están desarrollando distintos modelos educativos para que cada centro educativo gracias a su autonomía elija la mejor opción de acuerdo con el tipo de enseñanzas que ofertan y los distintos niveles educativos que desarrollan. El objetivo del presente estudio ha sido desarrollar un instrumento válido y fiable para evaluar la práctica docente no presencial en Formación Profesional. El enfoque metodológico corresponde a una investigación por encuesta transversal de carácter cuantitativo. Se ofrecen los diferentes resultados de los valores de alfa de Cronbach, así como una serie de estadísticos descriptivos. La estructura factorial de esta escala es analizada mediante análisis factorial exploratorio, análisis confirmatorio utilizando una muestra de 329 profesores. Los análisis realizados muestran que la escala se compone de seis factores: índice de actitud del alumnado-profesorado, atención a la diversidad, índice de coordinación docente, índice de eficacia virtual, índice de orientación y tutoría, índice de práctica docente virtual.

\section{PALABRAS CLAVE.}

Enseñanza, aprendizaje, evaluación, competencias.

\section{ABSTRACT.}

The COVID-19 pandemic seriously threatens the health of humanity, causing all types of consequences. The education sector is one of the most affected. Student learning has been reduced in the last quarter of the 2019-20 school year, and if science doesn't find a quick

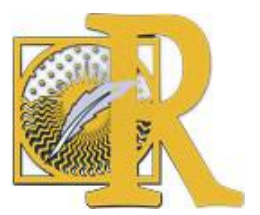

Fecha de recepción: 30-01-2021 Fecha de aceptación: 09-02-2021

Racero-Montes, F. J., Ramos-Corpa, M. J. \& Castillo-García, M. (2021). Validación de un instrumento de práctica docente no presencial en Formación Profesional

International Journal of Educational Research and Innovation (IJERI), 15, 444-459 ISSN: 2386-4303 DOI https://doi.org/10.46661/ijeri.5634 
solution to fight the virus, the teaching methods that have had to be forced during this time will likely have to be repeated over the next year. In this context, public administrations within the framework of their constitutional powers are developing different educational models so that each teaching center, thanks to its autonomy, chooses the best option according to the type of education they offer and the different educational levels they develop. The objective of this study has been to develop a valid and reliable instrument to evaluate teaching practice in times of pandemic. The methodological approach corresponds to a cross-sectional research survey of a quantitative nature. The different results of Cronbach's alpha values are offered, as well as a series of descriptive statistics. The factorial structure of this scale is analyzed by exploratory factor analysis, confirmatory analysis using a sample of 329 teachers. The studies carried out show that the scale is made up of six factors: student-teacher attitude index, attention to diversity, teacher coordination index, virtual efficiency index, orientation and tutoring index, virtual teaching practice index.

\section{KEY WORDS.}

Teaching, learning, evaluation, competency based teaching.

\section{Introducción.}

La práctica docente depende de multitud de variables; entre ellas se encuentran los conocimientos de cada docente, la motivación tanto del discente como del docente, las relaciones sociales, el nivel de partida del discente, los materiales curriculares, la familia, los objetivos que vienen marcados por la normativa vigente para cada uno de los niveles, etc. (Alanís, 2001; Sarramona 1998; Squires 2004). Destacamos una variable por excelencia respecto a las demás, la referida a la práctica docente, donde a su vez confluyen un conjunto de matices o subvariables que merecen ser atendidas por separado y que configuran el verdadero acto educativo. De igual forma, habrá de tenerse en cuenta los condicionantes marcados por los estilos cognitivos del alumnado, así como las estrategias y estilos docentes del profesorado. Al respecto, hay que decir, que si bien, las orientaciones metodológicas se establecen en el currículo de cada una las materias y áreas de cada etapa educativa, cada docente, en virtud del proyecto educativo elaborado por el centro, deberá configurar un método común que canalice el desempeño docente con el objetivo de facilitar el proceso educativo y aunar estrategias para alcanzar los objetivos y las competencias propias de cada etapa, en nuestro caso singular las referidas a la Formación Profesional.

En el marco actual, afectados por la pandemia COVID-19, nos encontramos con un escenario incierto, donde los procesos educativos se están viendo afectados por un nuevo paradigma de enseñanza-aprendizaje donde se están poniendo en valor los avances y las innovaciones educativas que estos años atrás se han podido implementar, dando como resultado algunas buenas prácticas en la docencia relacionada con las nuevas tecnologías. El impacto de la pandemia está generando nuevas formas de hacer educación, donde la crisis educativa podría revertirse en una oportunidad para generar conocimiento sobre los modos y formas en los que se puede enseñar y aprender. Al mismo tiempo es de importancia resaltar la posibilidad de generar una nueva brecha educativa en las familias más desfavorecidas, lo

Fecha de recepción: 30-01-2021 Fecha de aceptación: 09-02-2021

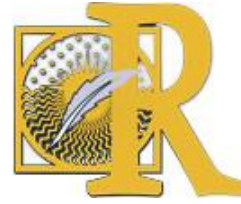

Racero-Montes, F. J., Ramos-Corpa, M. J. \& Castillo-García, M. (2021). Validación de un instrumento de práctica docente no presencial en Formación Profesional

International Journal of Educational Research and Innovation (IJERI), 15, 444-459 ISSN: 2386-4303 DOI https://doi.org/10.46661/ijeri.5634 
que hace pensar en la debilidad de la articulación del sistema cuando se generan situaciones que afectan a la presencialidad del acto docente.

De este modo, el sistema educativo se encuentra inmerso en una situación de incertidumbre, donde la presencia e interacción docente-discente, deja de ser relevante, utilizando nuevos canales de comunicación y nuevas formas de interrelación; ahora bien, dependiendo de la etapa educativa, este tipo de modelos en los centros docentes pudiera afectar de distinta forma. Nos proponemos diseñar y validar un instrumento para medir el proceso de enseñanza-aprendizaje en los centros educativos que desarrollan la etapa educativa de la Formación Profesional de forma no presencial y por tanto utilizando las tecnologías de la información (López, Pozo, Ávila, y Montero 2020).

Para poder realizar un diagnóstico de la práctica docente actual (COVID-19), es fundamental disponer de un instrumento de evaluación riguroso basado en cuestionarios, que aleje tanto los prejuicios como la subjetividad, cumpliendo con unos criterios mínimos de fiabilidad y validez (Furlong, Morrison, Cornell, y Skiba, 2004).

Respecto a los referentes previos de investigación en el marco de los diseños de evaluación de instrumentos son muchos y destacados: Cava, Povedano, Buelga y Musitu (2015); Agreda, Hinojo y Sola (2016); Dorantes, Hernández y Tobón (2016); Cava y Buelga (2018); Lara, Saracostti, Navarro, de Toro, Miranda-Zapata, Trigger, y Fuster (2018); Ariza y Muñoz (2020); Muñoz González, Hidalgo Ariza y Marín Díaz (2020); Santos, Cañadas, Martínez y García Rico (2020). No obstante respecto a situaciones como las vividas en la actualidad es incuestionable que no se han detectado trabajos hasta el momento, por lo que nos proponemos como objetivo el proporcionar una herramienta fiable para medir los efectos de la pandemia en el aprendizaje de los estudiantes de Formación Profesional.

\subsection{Formación Profesional.}

Nos encontramos ante un nuevo escenario de construcción de un macrosistema de Formación Profesional en el que cada país debe dar respuesta ante la nueva situación social, política, económica y por ende educativa. Son muchos los intentos de aunar esfuerzos por establecer criterios que den cierta homogeneidad en la Formación Profesional; en Europa, el Centro Europeo para el desarrollo de la Formación Profesional (Cedefop), organiza eventos y proyectos de investigación para desarrollar conocimiento y establecer líneas de actuación. Los distintos países se encuentran en pleno proceso de rediseño de cada sistema, tomando como referencia alguno de los modelos actuales; modelo liberal de mercado (Gran Bretaña), modelo regulado por el Estado (Francia) y el modelo corporativo y dual (Alemania). En España, hace algún tiempo que se incorporó el modelo de Formación Profesional dual donde el conjunto de competencias de cada ciclo formativo se alcanza en escenarios reales de trabajo.

En este sentido, los países miembros de los diferentes estados realizan a nivel nacional estudios y trabajos a través de grupos de trabajo para reflexionar sobre los nuevos subsistemas afectados por las variables sociales, económicas y educativas que inciden en los procesos educativos, tanto a nivel de diseño de nuevos programas, como del análisis de la situación general de los trabajadores capacitados en relación con la sobrecualificación existente. Merece la pena reseñar los estudios realizados por el Observatorio de la Formación

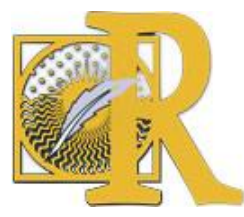

Fecha de recepción: 30-01-2021 Fecha de aceptación: 09-02-2021

Racero-Montes, F. J., Ramos-Corpa, M. J. \& Castillo-García, M. (2021). Validación de un instrumento de práctica docente no presencial en Formación Profesional

International Journal of Educational Research and Innovation (IJERI), 15, 444-459 ISSN: 2386-4303 DOI https://doi.org/10.46661/ijeri.5634 
Profesional en España, en el que se hace constar que el 68,8\% de las personas que cuentan con un Ciclo Formativo de Grado Superior se encuentran desarrollando ocupaciones de menor nivel, de ahí la necesidad de incrementar la formación profesional de grado medio, no obstante, la solución no es fácil, como así muestra el citado informe "La principal vía para corregir el desajuste por sobrecualificación pasa por aumentar el peso de las ocupaciones de alta cualificación en la estructura productiva. Esta es una tarea compleja cuya solución procede, principalmente, del cambio en el tipo de demanda por parte de los empleadores" (Gamboa, Moso, Albizu, Lafuente, Mondaca, Murciego et al. 2020, p.21).

Es pertinente señalar que en la nueva Ley Orgánica 3/2020 de 29 de diciembre de modificación de la Ley Orgánica de Educación (LOMLOE) se hace referencia al nuevo sistema de la Formación Profesional en España, donde se modifica la actual Formación Profesional Básica, denominándose Ciclo Formativo de Grado Básico. El preámbulo de la nueva ley lo contempla de esta forma "La formación profesional es también objeto de varias revisiones, con dos finalidades; una de ellas, la mejora en el reconocimiento social de los itinerarios formativos de formación profesional, para aproximarnos a las tasas de alumnado que opta por esta vía en el resto de los países europeos; la segunda, la flexibilización de las enseñanzas y la agilización de los procesos de incorporación de nuevos contenidos. Para ello, por un lado, se establece que comprende los ciclos formativos de grado básico, de grado medio y de grado superior, así como los cursos de especialización" (BOE núm. 340 de 2020, p. 122876).

De este modo, además de las variables contextuales advertidas por la nueva situación COVID-19, el profesorado de la Formación Profesional, habrá de tener presente la complejidad del acto docente, multidimensional, donde el desarrollo científico-técnico del momento y la situación actual habrá de complementarse con los factores sociales, que hacen del momento enfrentarse a retos telemáticos donde enseñar y aprender se alejan de los escenarios convencionales, para pasar a nuevas relaciones telemáticas. Por tanto, la naturaleza social del acto pierde intensidad y toman protagonismo otras competencias relacionales donde lo importante sea, no ya el capacitar al alumnado, sino que alcancen competencia de la búsqueda de información actualizada ante los problemas sobrevenidos en un momento y situación específica, por ello se tiende al aprendizaje eficiente de los nuevos conocimientos (Ronquillo, Cabrera y Barberán, 2019).

Conviene destacar de igual forma, que los escenarios de aprendizaje deben modificar las metodologías y las propias competencias del profesorado; en este sentido no todo profesional de la enseñanza dispone los perfiles de competencia idóneos para cualquier nivel, máxime de una enseñanza tan específica como la Formación Profesional "suele ser muy habitual pensar en el docente desde la óptica del propio sistema educativo pensando con ello en un profesional que desarrolla su actividad en los distintos niveles y que cuenta con una formación normalmente ligada a la Facultad de Ciencias de la Educación, cuando en realidad son múltiples las situaciones y escenarios de actuación profesional tanto dentro como fuera del sistema y a la vez múltiples los perfiles que podemos encontrar dentro de lo que podemos considerar la familia profesional de la educación, así como la formación y profesionalización de los mismos" (Tejada, 2008:4). En este sentido el contexto dual formación teórico práctica en escenarios meramente educativos habrá de complementarse con los escenarios

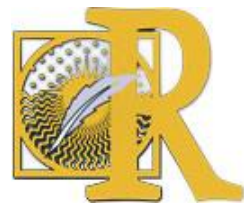

Fecha de recepción: 30-01-2021 Fecha de aceptación: 09-02-2021

Racero-Montes, F. J., Ramos-Corpa, M. J. \& Castillo-García, M. (2021). Validación de un instrumento de práctica docente no presencial en Formación Profesional

International Journal of Educational Research and Innovation (IJERI), 15, 444-459 ISSN: 2386-4303 DOI https://doi.org/10.46661/ijeri.5634 
productivos en interrelación con profesionales alejados del escenario educativo, por lo que habrá de definirse el entronque centro formativo reglado-empresa "La conexión entre las enseñanzas de formación profesional y el mercado laboral implica una mayor colaboración con las empresas que permita adaptar estas enseñanzas a la demanda laboral. Fomentar la innovación, favorecer el desarrollo de las competencias transversales y potenciar el emprendimiento son claves que marcan la estrategia para que estas enseñanzas propicien las destrezas que posibiliten afrontar nuevos retos" (BOCM. núm. 170 de 2019, p. 11)

\section{Material y métodos.}

\subsection{Objetivos.}

El objetivo del presente estudio ha sido desarrollar un instrumento "ad hoc" válido y fiable para evaluar la práctica docente no presencial en Formación Profesional, denominado "Escala de Medición de Docencia No Presencial en Formación Profesional" (en adelante, Escala FP DNP). La validación se realizó a partir del confinamiento domiciliario surgido por la pandemia COVID-19". El cuestionario, parte de la revisión bibliográfica de estudios e investigaciones sobre la temática, teniendo en cuenta, la ausencia de investigaciones al respecto previas con las que contamos. Por ello, entendemos una oportuna revisión de estudios de inicio para valorar y contribuir a la mejora de los procesos de enseñanzaaprendizaje en circunstancias a las vividas en los momentos actuales. El enfoque metodológico corresponde a una investigación por encuesta transversal de carácter cuantitativo. Para el desarrollo de la construcción del instrumento se han seguido las siguientes fases:

a) Identificación de dimensiones: en relación con el objetivo propuesto, se procedió a una profunda revisión de todo el marco bibliográfico y estudios de investigación próximos a la investigación iniciada, concluyendo en seis dimensiones para la escala FP DNP (véase Tabla 1).

b) Redacción de ítems: una vez discriminados los factores o dimensiones que intervendrían en el estudio, se procedió a redactar los ítems. El cuestionario original estaba compuesto por 30 ítems con una escala tipo Likert admitiendo cuatro grados (1. Nada de acuerdo, 2. Algo de acuerdo, 3. Bastante de acuerdo, 4. Totalmente de acuerdo)

c) Validación: para corroborar la validez de contenido y estructura se sometió a un procedimiento de juicio de expertos, definido como una opinión informada de personas con amplia trayectoria en el tema, que son reconocidas por otros como expertos (Escobar-Pérez y Cuervo-Martínez, 2008). Se envió tanto el cuestionario como el objetivo de la investigación a cinco inspectores de educación, con gran experiencia y conocimiento en los aspectos relacionados con las distintas categorías. Los criterios de evaluación fueron los de pertinencia, suficiencia, coherencia, síntesis y semántica.

d) Ajuste del cuestionario: en función de las distintas puntuaciones, se procedió a la reformulación de algunos ítems para evitar posibles problemas en su comprensión. Se eliminaron 3 ítems por subsumir a otros ya incluidos, además de no ser pertinentes 
en la selección de información. El cuestionario final se compuso de 27 ítems. Quedó redactado en formato digital para ser enviado a través de la herramienta de generación de formularios de Google, abarcando toda la comunidad autónoma andaluza.

e) Administración del cuestionario. Se creó una base de datos con todos los correos electrónicos de los centros educativos de Andalucía, proporcionando el cuestionario de forma masiva a toda la población docente de la comunidad autónoma, con copia oculta para preservar el anonimato y protección de los datos de los participantes del estudio.

Tabla 1.

Dimensiones e ítems de la escala.

Fuente: Elaboración propia a partir del (art.91) de la LOE.

\begin{tabular}{|c|c|c|}
\hline Dimensión & & Ítems \\
\hline \multirow{4}{*}{$\begin{array}{l}\text { IAA: índice de actitud del } \\
\text { alumnado-profesorado. }\end{array}$} & 1 & Puede atender a todo el alumnado que necesite refuerzo en alguna materia. \\
\hline & 2 & $\begin{array}{l}\text { El comportamiento del alumnado a través de la enseñanza virtual es mejor que en el } \\
\text { aula ordinaria, reduciéndose las correcciones que se imponen. }\end{array}$ \\
\hline & 3 & El alumnado hace las tareas y actividades y las envía en tiempo y forma. \\
\hline & 4 & $\begin{array}{l}\text { Propone tareas y actividades para que el alumnado pueda realizarlas de forma individual } \\
\text { fuera del tiempo escolar. }\end{array}$ \\
\hline \multirow{3}{*}{$\begin{array}{l}\text { IAD: índice de atención a } \\
\text { la diversidad. }\end{array}$} & 5 & Puede atender a todo el alumnado con dificultades en el aprendizaje. \\
\hline & 6 & $\begin{array}{l}\text { El alumnado desarrolla más habilidades sociales en la enseñanza virtual que en la } \\
\text { enseñanza presencial. }\end{array}$ \\
\hline & 7 & Los alumnos con NEE son atendidos en la enseñanza virtual. \\
\hline \multirow{4}{*}{$\begin{array}{l}\text { ICD: } \quad \text { índice } \\
\text { coordinación docente. }\end{array}$} & 8 & La dirección del centro apoya y coordina la enseñanza virtual del centro. \\
\hline & 9 & $\begin{array}{l}\text { Se realizan Claustros de Profesores utilizando medios tecnológicos, con la participación } \\
\text { de todos los integrantes. }\end{array}$ \\
\hline & 10 & $\begin{array}{l}\text { Se realizan Equipos de Ciclo/Reuniones de Departamento, utilizando medios } \\
\text { tecnológicos, con la participación de todos los integrantes. }\end{array}$ \\
\hline & 11 & $\begin{array}{l}\text { La hora de clase dispone de un tiempo de explicación, otro de trabajo del alumnado y } \\
\text { otro de análisis y evaluación de actividades. }\end{array}$ \\
\hline \multirow{6}{*}{$\begin{array}{l}\text { IEV: índice de eficacia } \\
\text { virtual. }\end{array}$} & 12 & Ha modificado la planificación curricular que tenía. \\
\hline & 13 & $\begin{array}{l}\text { Las explicaciones de los contenidos utilizando la enseñanza virtual tienen la misma } \\
\text { eficacia que en la docencia presencial. }\end{array}$ \\
\hline & 14 & Tiende a utilizar recursos flexibles, variados y accesibles a la totalidad del alumnado. \\
\hline & 15 & $\begin{array}{l}\text { El alumnado es capaz de comunicarse de forma oral con soltura a través de enseñanza } \\
\text { virtual. }\end{array}$ \\
\hline & 16 & Hay mayor interacción alumno-profesor en la enseñanza virtual. \\
\hline & 17 & Los objetivos de cada unidad se alcanzan con la enseñanza virtual. \\
\hline \multirow[t]{3}{*}{$\begin{array}{l}\text { IOT: índice de orientación } \\
\text { y tutoría. }\end{array}$} & 18 & $\begin{array}{l}\text { La evaluación se hace principalmente a través de la observación y participación en clase } \\
\text { y los trabajos entregados. }\end{array}$ \\
\hline & 19 & $\begin{array}{l}\text { Se ha potenciado la coordinación de los órganos de coordinación (Equipos de } \\
\text { Ciclo/Reuniones de Departamento) para unificar tareas, evaluación, atención al } \\
\text { alumnado... }\end{array}$ \\
\hline & 20 & Los tutores recogen las quejas de los padres/madres. \\
\hline \multirow{3}{*}{$\begin{array}{l}\text { IPDV: índice de práctica } \\
\text { docente virtual. }\end{array}$} & 21 & Puede realizar un control de asistencia del alumnado al inicio de clase. \\
\hline & 22 & $\begin{array}{l}\text { Inicia un primer contacto con el alumnado para verificar la conexión a la plataforma } \\
\text { virtual. }\end{array}$ \\
\hline & 23 & La Jefatura de Estudios está coordinando todo el proceso educativo del alumnado. \\
\hline
\end{tabular}

Fecha de recepción: 30-01-2021 Fecha de aceptación: 09-02-2021

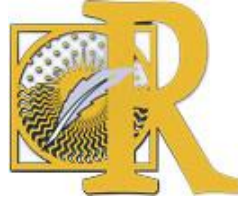

Racero-Montes, F. J., Ramos-Corpa, M. J. \& Castillo-García, M. (2021). Validación de un instrumento de práctica docente no presencial en Formación Profesional

International Journal of Educational Research and Innovation (IJERI), 15, 444-459

ISSN: 2386-4303 DOI https://doi.org/10.46661/ijeri.5634 


\subsection{Muestra y procedimiento.}

La muestra estaba constituida por un total de 329 profesores y profesoras distribuidos por las 8 provincias andaluzas. Para el análisis de la fiabilidad y validez estadística del instrumento se utilizó el programa SPSS, versión 21.0 para Windows. El análisis realizado consta de un análisis factorial exploratorio (AFE), de cuyo resultado se eliminaron finalmente 4 ítems de los 27 totales, quedando el cuestionario compuesto por 23 ítems (véase Tabla 1). Posteriormente se realizó un análisis factorial confirmatorio (AFC), analizándose la bondad de los datos obtenidos con el programa AMOS.16. En primer lugar, se detectaron valores perdidos o fuera de escala, $u$ otros por los que no deba aplicarse el AFC (normalidad multivariante, relaciones lineales entre variables, ausencia de multicolinealidad, etc.). Posteriormente el modelo fue ajustado a partir del examen de los residuos estandarizados, índices de modificación, etc. Para determinar el ajuste se han utilizado los índices más habituales: X2/grados de libertad, AGFI, CFI, RMSEA, NFI, NNFI, GFI e IFI.

\section{Resultados.}

En primer lugar, se realizó un análisis de la fiabilidad correspondiente a la consistencia y/o estabilidad de las medidas, para determinar si el cuestionario es capaz de medir lo que se pretende evaluar. La fiabilidad se calculó por medio de la consistencia interna de las dimensiones planteadas. Teniendo en cuenta las consideraciones de George y Mallery (2003) que establecieron como nivel aceptable un coeficiente de Alfa de Cronbach >,7. Del análisis de los datos se obtiene que tanto las dimensiones de forma independiente, como el conjunto del cuestionario supera el citado nivel de aceptabilidad (véase Tabla 2). También se realizó la prueba de las dos mitades de Guttman obteniéndose un valor de 0,791 (véase Tabla 3).

\section{Tabla 2.}

Coeficiente Alfa del Cronbach de las dimensiones de la escala FP DNP.

Fuente: Elaboración propia.

\begin{tabular}{|l|c|}
\hline \multicolumn{1}{|c|}{ Dimensión } & Coeficiente Alfa de Cronbach \\
\hline $\begin{array}{l}\text { IAAP: índice de actitud del alumnado- } \\
\text { profesorado. }\end{array}$ & 0,742 \\
\hline IAD: índice de atención a la diversidad. & 0,811 \\
\hline ICD: índice de coordinación docente. & 0,739 \\
\hline IEV: índice de eficacia virtual. & 0,861 \\
\hline IOT: índice de orientación y tutoría. & 0,744 \\
\hline $\begin{array}{l}\text { IPDV: índice de práctica docente } \\
\text { virtual. }\end{array}$ & 0,722 \\
\hline TOTAL & 0,887 \\
\hline
\end{tabular}

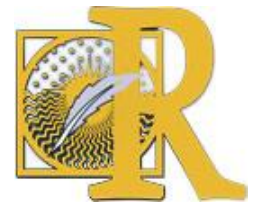

Fecha de recepción: 30-01-2021 Fecha de aceptación: 09-02-2021

Racero-Montes, F. J., Ramos-Corpa, M. J. \& Castillo-García, M. (2021). Validación de un instrumento de práctica docente no presencial en Formación Profesional 
Tabla 3.

Estadístico de fiabilidad de las dos mitades de Guttman de la escala FP DNP. Fuente: Elaboración propia.

\begin{tabular}{|c|c|c|c|}
\hline \multirow[t]{5}{*}{ Alfa de Cronbach } & \multirow[t]{2}{*}{ Parte 1} & Valor & 850 \\
\hline & & $\mathrm{N}$ de elementos & $12 a$ \\
\hline & \multirow[t]{2}{*}{ Parte 2} & Valor & 822 \\
\hline & & $\mathrm{N}$ de elementos & $11 \mathrm{~b}$ \\
\hline & \multicolumn{2}{|c|}{$\mathrm{N}$ total de elementos } & 23 \\
\hline \multicolumn{3}{|l|}{ Correlación entre formularios } & 656 \\
\hline \multirow{2}{*}{$\begin{array}{l}\text { Coeficiente de Spearman- } \\
\text { Brown }\end{array}$} & \multicolumn{2}{|c|}{ Longitud igual } & ,793 \\
\hline & \multicolumn{2}{|c|}{ Longitud desigual } &, 793 \\
\hline \multicolumn{3}{|c|}{ Coeficiente de dos mitades de Guttman } &, 791 \\
\hline
\end{tabular}

Antes de iniciar el análisis factorial se procedió a calcular los valores de las pruebas de KMO (Kaiser, Meyer y Olkin) y Bartlett para determinar si el número de datos es el adecuado para el análisis que se pretende abordar. En este sentido, cuanto más cercano a la unidad sea el valor del coeficiente de KMO mayor bondad presentará el instrumento, si el valor del test de esfericidad de Bartlett es menor de ,05 es adecuado y pertinente realizar el análisis factorial. Se obtuvo un valor del coeficiente de $\mathrm{KMO}$ de ,882, el test de esfericidad de Bartlett arrojó los siguientes valores: aproximación Chi-cuadrado de 3476,215; grados de libertad: 253 y una significación de ,000.

EI AFE, nos permitió comparar la estructura teórica que habíamos planteado con la que subyace del instrumento, facilitando una información importante para el estudio de la validez de constructo, y por tanto mejorando el cuestionario. Como se muestra en la Tabla 4 la extracción de factores mediante el análisis de componentes principales y rotación Varimax arrojó 6 factores primarios que explicaban el 65, 875\% acumulado de la varianza total, oscilando las comunalidades entre 0,417 del ítem 8 y 0,826 del ítem 5.

Tabla 4.

Varianza total explicada por el modelo.

Fuente: Elaboración propia.

\begin{tabular}{|c|c|c|c|c|c|c|}
\hline Componente & \multicolumn{3}{|c|}{ Autovalores iniciales } & \multicolumn{3}{c|}{$\begin{array}{c}\text { Sumas de cargas al cuadrado de la } \\
\text { extracción }\end{array}$} \\
\cline { 2 - 6 } & Total & $\begin{array}{c}\text { \% de } \\
\text { varianza }\end{array}$ & $\begin{array}{c}\text { \% } \\
\text { acumulado }\end{array}$ & Total & $\begin{array}{c}\text { \% de } \\
\text { varianza }\end{array}$ & $\begin{array}{c}\% \\
\text { acumulado }\end{array}$ \\
\hline 1 & 7,342 & 31,924 & 31,924 & 7,342 & 31,924 & 31,924 \\
\hline 2 & 2,811 & 12,223 & 44,146 & 2,811 & 12,223 & 44,146 \\
\hline 3 & 1,473 & 6,405 & 50,552 & 1,473 & 6,405 & 50,552 \\
\hline 4 & 1,371 & 5,960 & 56,512 & 1,371 & 5,960 & 56,512 \\
\hline 5 & 1,150 & 4,998 & 61,510 & 1,150 & 4,998 & 61,510 \\
\hline 6 & 1,004 & 4,365 & 65,875 & 1,004 & 4,365 & 65,875 \\
\hline
\end{tabular}

Por medio de los factores rotados de la matriz y el peso factorial de cada uno de los ítems (véase Tabla 5) se observa que todos los ítems presentan cargas superiores a 0,4 y que están agrupados según las dimensiones establecidas con anterioridad.

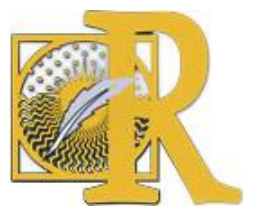

Fecha de recepción: 30-01-2021 Fecha de aceptación: 09-02-2021

Racero-Montes, F. J., Ramos-Corpa, M. J. \& Castillo-García, M. (2021). Validación de un instrumento de práctica docente no presencial en Formación Profesional

International Journal of Educational Research and Innovation (IJERI), 15, 444-459 ISSN: 2386-4303 DOI https://doi.org/10.46661/ijeri.5634 
Tabla 5.

Matriz de factores rotados.

Fuente: Elaboración propia.

\begin{tabular}{|c|c|c|c|c|c|c|}
\hline & \multicolumn{6}{|c|}{ Componentes } \\
\hline & IAAP & IAD & $I C D$ & IEV & IOT & IPDV \\
\hline 11 & ,485 & & & & & \\
\hline 12 & ,819 & & & & & \\
\hline 13 &, 721 & & & & & \\
\hline 14 &, 564 & & & & & \\
\hline 15 & & 831 & & & & \\
\hline 16 & & 853 & & & & \\
\hline 17 & & 572 & & & & \\
\hline 18 & & & ,492 & & & \\
\hline 19 & & & 825 & & & \\
\hline 110 & & & 822 & & & \\
\hline 111 & & & 712 & & & \\
\hline 112 & & & & 802 & & \\
\hline 113 & & & & ,767 & & \\
\hline 114 & & & & 613 & & \\
\hline 115 & & & & 681 & & \\
\hline 116 & & & & 641 & & \\
\hline 117 & & & & ,720 & & \\
\hline 118 & & & & & ,839 & \\
\hline 119 & & & & & ,800 & \\
\hline 120 & & & & & ,759 & \\
\hline 121 & & & & & & 826 \\
\hline 122 & & & & & & ,798 \\
\hline 123 & & & & & & ,614 \\
\hline
\end{tabular}

Para contrastar la estructura del modelo obtenido con AFE, se llevó a cabo un AFC, utilizando como método de estimación Máxima Verosimilitud, obteniendo los resultados expuestos en la Figura 1. 


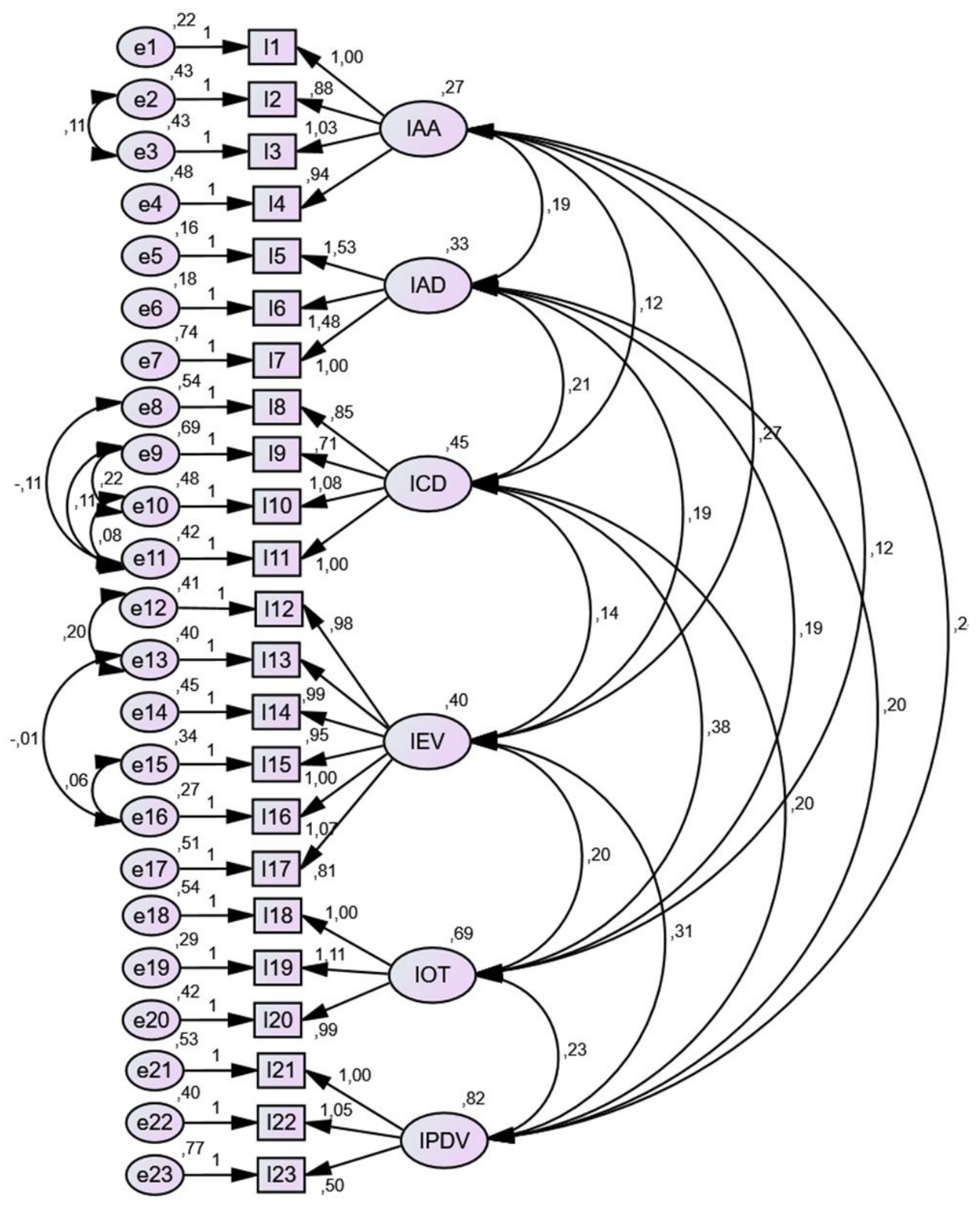

Figura1.

Modelo de seis factores (AFC).

Para ver la bondad del ajuste del modelo planteado se ha considerado la prueba $x^{2} /$ grados de libertad, y los índices AGFI, CFI, RMSEA, NFI, NNFI, GFI e IFI. La Tabla 6 muestra los índices de ajuste para el modelo de investigación, junto con los valores sugeridos. Los resultados manifiestan que el modelo de investigación tiene un ajuste aceptable sobre el límite mínimo / máximo. definido (Lei, P.W., 2009).

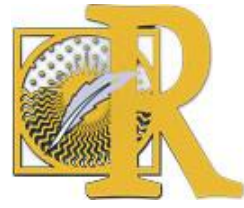


Tabla 6.

Índices de ajuste del modelo.

Fuente: Elaboración propia.

\begin{tabular}{|l|c|c|}
\hline \multicolumn{1}{|c|}{ Indicador } & Resultado & $\begin{array}{l}\text { Valor } \\
\text { recomendado }\end{array}$ \\
\hline $\mathrm{X}^{2}$ /grados de libertad & 1,701 & $\leq 3,00$ \\
\hline Normed Fit Index (NFI) & 0,901 & $\geq 0,90$ \\
\hline Nonnormed Fit Index (NNFI) & 0,947 & $\geq 0,90$ \\
\hline Comparative Fit Index (CFI) & 0,956 & $\geq 0,90$ \\
\hline Adjusted Goodness-of-Fit Index (AGFI) & 0,887 & $\geq 0,80$ \\
\hline $\begin{array}{l}\text { Root Mean Squeare Error of Approximation } \\
\text { (RMSEA) }\end{array}$ & 0,046 & $\leq 0,05$ \\
\hline Goodness-of-Fit Index (GFI) & 0,915 & $\geq 0,90$ \\
\hline Incremental Fit Index (IFI) & 0,957 & $\geq 0,90$ \\
\hline
\end{tabular}

\subsection{Análisis correlacional.}

Se ha desarrollado el análisis correlacional de las dimensiones del cuestionario. En la Tabla 7 se muestran los datos obtenidos al realizar la prueba de correlación de Pearson, con el objeto de observar la relación existente entre las dimensiones del cuestionario.

De los resultados obtenidos se puede concluir que existe una relación entre las dimensiones del cuestionario al ser la relación bilateral entre los distintos factores significativa a nivel de 0,01 .

\section{Tabla 7.}

Correlación entre dimensiones.

Fuente: Elaboración propia.

\begin{tabular}{|c|c|c|c|c|c|c|c|}
\hline & & IAAP & IAD & ICD & IEV & IOT & IPDV \\
\hline \multirow[t]{3}{*}{ IAAP } & Correlación de Pearson & 1 &, $484^{\star \star}$ & $226^{\star \star}$ & $612^{\star *}$ & $217^{* \star}$ &, $385^{\star \star}$ \\
\hline & Sig. (bilateral) & &, 000 &, 000 &, 000 &, 000 &, 000 \\
\hline & $\mathrm{N}$ & 329 & 329 & 329 & 329 & 329 & 329 \\
\hline \multirow[t]{3}{*}{ IAD } & Correlación de Pearson & $484^{\star \star}$ & 1 &, $426^{\star \star}$ & $472^{\star *}$ & ,419** & $351^{* \star}$ \\
\hline & Sig. (bilateral) &, 000 & &, 000 &, 000 &, 000 & ,000 \\
\hline & $\mathrm{N}$ & 329 & 329 & 329 & 329 & 329 & 329 \\
\hline \multirow[t]{3}{*}{ ICD } & Correlación de Pearson & $226^{\star *}$ &, $426^{\star *}$ & 1 & ,249** &, $526^{* *}$ &, $246^{\star *}$ \\
\hline & Sig. (bilateral) &, 000 &, 000 & &, 000 &, 000 &, 000 \\
\hline & $\mathrm{N}$ & 329 & 329 & 329 & 329 & 329 & 329 \\
\hline \multirow[t]{3}{*}{ IEV } & Correlación de Pearson & $612^{\star \star}$ & $472^{\star \star}$ & ,249** & 1 & $310^{\star \star}$ & $455^{\star *}$ \\
\hline & Sig. (bilateral) &, 000 &, 000 &, 000 & &, 000 &, 000 \\
\hline & $\mathrm{N}$ & 329 & 329 & 329 & 329 & 329 & 329 \\
\hline \multirow[t]{3}{*}{ IOT } & Correlación de Pearson & $217^{* *}$ & $419^{* *}$ &, $526^{* *}$ & $310^{* *}$ & 1 & $258^{\star \star}$ \\
\hline & Sig. (bilateral) &, 000 &, 000 &, 000 &, 000 & &, 000 \\
\hline & $\mathrm{N}$ & 329 & 329 & 329 & 329 & 329 & 329 \\
\hline \multirow[t]{3}{*}{ IPDV } & Correlación de Pearson & $385^{\star \star}$ & $351^{\star *}$ & $246^{\star \star}$ & $455^{\star *}$ & $258^{* \star}$ & 1 \\
\hline & Sig. (bilateral) &, 000 &, 000 &, 000 &, 000 &, 000 & \\
\hline & $\mathrm{N}$ & 329 & 329 & 329 & 329 & 329 & 329 \\
\hline
\end{tabular}




\section{Discusión.}

El objetivo de este estudio fue el de diseñar y analizar las propiedades y la estructura factorial de un instrumento para medir el proceso de enseñanza-aprendizaje no presencial en los centros educativos que desarrollan la etapa educativa de la Formación Profesional. Ha sido aplicada a una muestra muy significativa de la población de centros de Andalucía donde se imparten este tipo de enseñanzas, llegando a la conclusión de que el mismo es fiable debido a la alta consistencia interna tanto en la escala general como en cada una de sus dimensiones.

Del mismo modo, los análisis correlacionales aplicados muestran una alta interrelación entre sus seis dimensiones o factores; actitud del alumnado-profesorado, atención a la diversidad, coordinación docente, eficacia virtual, orientación y tutoría, práctica docente virtual. Todos estos factores tienen una alta incidencia desde el punto de vista teórico, así en trabajos de investigación sobre buenas prácticas en la educación, Lizasoain y Angulo (2014), establecen catorce factores o categorías de buenas prácticas; proyectos, planes y formación; metodologías y materiales de enseñanza; atención a la diversidad; seguimiento del alumnado; atención individualizada; orientación y tutoría; evaluación del alumnado; gestión del tiempo; liderazgo y equipo directivo; modelos de gestión y organización; coordinación; implicación y pertenencia al centro; evaluación de los docentes, del propio centro, de programas y actividades y uso de la evaluación diagnóstica; clima y convivencia; imagen, instalaciones y recursos; familia, comunidad y capital social. Por otro lado, Ramos y Castillo (2013) establecieron cinco factores o categorías en un trabajo de investigación: organización, gestión y dirección escolar (Castillo, Ramos y Del Moral 2020); docencia del profesorado; orientación, tutoría, convivencia y relaciones con las familias; atención a la diversidad; utilización de las nuevas tecnologías. Dada la preocupante situación educativa, entendemos que este tipo de estudios deberían incrementarse en futuras investigaciones generando conocimiento sobre la metodología que debería subyacer en relación con cada una de las dimensiones significativas a las que ha llegado el presente estudio.

Con referencia al modelo confirmatorio, quedó compuesto por seis dimensiones:

Dimensión "índice de actitud del alumnado-profesorado". Esta dimensión compuesta por un total de 4 ítems, ha permitido evaluar la necesidad que tiene el alumnado de ser atendido de forma diferenciada, en la que se le puedan aplicar planes de refuerzo educativo (LOE, artículo 71); por otro lado, se evalúa el comportamiento del alumnado a través de la enseñanza virtual, en la que deberían de reducirse las correcciones al alumnado con la mejora de la convivencia escolar (Ramos-Corpas, 2013). Del mismo modo se evalúa el nivel de compromiso del alumnado en relación a la realización de las tareas y actividades y las envía en tiempo y forma. Otro aspecto que mide es la práctica docente en relación con las tareas y actividades para que el alumnado pueda realizarlas de forma individual fuera del tiempo escolar (Coll, C. 2007; De la Torre, S. 2000).

Dimensión "atención a la diversidad". Esta dimensión compuesta por un total de 3 ítems, ha permitido evaluar si el profesorado puede atender a todo el alumnado con dificultades en el aprendizaje (LOE, artículo 71), si desarrolla más habilidades sociales en la enseñanza virtual que en la enseñanza presencial; aspecto novedoso en las formas de socialización escolar,

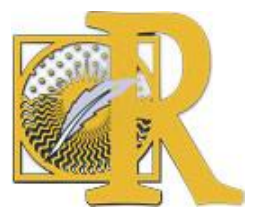

Fecha de recepción: 30-01-2021 Fecha de aceptación: 09-02-2021

Racero-Montes, F. J., Ramos-Corpa, M. J. \& Castillo-García, M. (2021). Validación de un instrumento de práctica docente no presencial en Formación Profesional

International Journal of Educational Research and Innovation (IJERI), 15, 444-459 ISSN: 2386-4303 DOI https://doi.org/10.46661/ijeri.5634 
ya que aparece un tipo de alumnado que es capaz de interaccionar más a través de lo digital que a través de la presencialidad (Castañeda, del Moral y Suárez, 2017). El último ítem es el referido a la atención de alumnos NEE (Necesidades Educativas Especiales) a través de la enseñanza virtual. No debería ser un hándicap la tecnología educativa y los medios de instrucción para la atención de este tipo de alumnado. Constituye un deber del centro educativo y por otro un derecho del alumnado, así se establece entre los derechos del alumnado "a recibir las ayudas y los apoyos precisos para compensar las carencias y desventajas de tipo personal, familiar, económico, social y cultural, especialmente en el caso de presentar necesidades educativas especiales, que impidan o dificulten el acceso y la permanencia en el sistema educativo" (LODE, artículo 6.h).

Dimensión "índice de coordinación docente.". Esta dimensión compuesta por un total de 4 ítems, ha permitido evaluar si el profesorado utiliza tanto en la planificación como en actividad docente los recursos TIC. En principio antes de la pandemia, cada profesor tenía un marco planificador de su proceso educativo a través de unidades temáticas encuadradas en las distintas programaciones didácticas teniendo en cuenta por un lado las competencias profesionales de cada módulo y los elementos del proyecto educativo. Una vez se modificó el modelo de enseñanza a la no presencialidad, el profesorado tuvo que modificar sus patrones de docencia para adaptarse a la nueva situación (Cabero, Fernández y Marín, 2017). Este índice evalúa variables de coordinación como la realización de reuniones del profesorado con el uso de TIC, así como otro conjunto de reuniones preceptivas en los centros escolares para poder desarrollar un Proyecto Educativo común. Igualmente aporta información sobre el apoyo y coordinación que ejerce la dirección en la enseñanza virtual del centro.

Dimensión "índice de eficacia virtual”. Esta dimensión compuesta por un total de 6 ítems, ha permitido evaluar si el profesorado ha modificado la planificación curricular que tenía antes de la situación COVID-19 (Perrenoud, 2004), y por otro lado la eficacia de su enseñanza, así como la interacción alumno-profesor en la enseñanza virtual, alcance de los objetivos propuestos, recursos utilizados y la capacidad de comunicarse de forma oral con soltura a través de enseñanza virtual. El profesorado deberá ser conocedor de las distintas plataformas virtuales (Canales y Marqués, 2007) realizaron estudios sobre la relación entre las nuevas tecnologías y los resultados escolares en distintos ejes (estrategias cognitivas, metacognitivas, de estímulo en el aprendizaje, de integración de competencias claves, etc.) Dimensión "índice de orientación y tutoría.". Esta dimensión compuesta por un total de 3 ítems, ha permitido evaluar si el profesorado realiza la evaluación se hace principalmente a través de la observación y participación en clase y los trabajos entregados (Castillo y Cabrerizo, 2007), si se ha potenciado la coordinación de los órganos de coordinación (reuniones de departamento) para unificar tareas, evaluación, atención al alumnado y por último la labor de los tutores. Téngase en cuenta que en la Formación Profesional aparecen distintos tutores, por un lado, el educativo del centro escolar y por otro el laboral cuando realiza la formación en centros de trabajo (FCT) o en los modelos de Formación Profesional Dual.

Dimensión "índice de práctica docente virtual". Esta dimensión compuesta por un total de 3 ítems, ha permitido evaluar si el profesorado realiza el control de asistencia del alumnado al

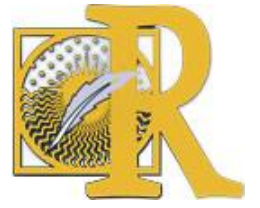

Fecha de recepción: 30-01-2021 Fecha de aceptación: 09-02-2021

Racero-Montes, F. J., Ramos-Corpa, M. J. \& Castillo-García, M. (2021). Validación de un instrumento de práctica docente no presencial en Formación Profesional

International Journal of Educational Research and Innovation (IJERI), 15, 444-459 ISSN: 2386-4303 DOI https://doi.org/10.46661/ijeri.5634 
inicio de clase, si inicia un primer contacto con el alumnado para verificar la conexión a la plataforma virtual y si la Jefatura de Estudios está coordinando todo el proceso educativo del alumnado. Estos tres vectores son factores claves influyentes en el normal desenvolvimiento de la clase, encontrándose entre las tareas que dodo profesor debe de realizar. Tareas que Marqués (2000) se refiere como las "tareas nucleares de un docente", resumidas en una serie de dimensiones; entre ellas la gestora y de control.

En definitiva, el cuestionario "Escala de Medición de Docencia No Presencial en Formación Profesional" resulta una herramienta válida y fiable para evaluar la práctica docente en los aspectos relacionados con la actitud del alumnado-profesorado, atención a la diversidad, coordinación docente, eficacia virtual, orientación, tutoría y práctica docente virtual.

Para finalizar consideramos necesario manifestar como limitación principal que el estudio se ha realizado íntegramente en la Comunidad Autónoma de Andalucía, teniendo en cuenta que en otras comunidades autónomas se desarrollan diferentes tipos de plataformas educativas que plantean diferencias en el desarrollo de la práctica docente. En futuras investigaciones, pretendemos utilizar el instrumento desarrollado, en el presente artículo, en ámbitos geográficos más amplios, para poder conocer cómo se desarrollan las seis dimensiones del instrumento en otras realidades docentes.

\section{REFERENCIAS.}

- Agreda Montoro, M., Hinojo Lucena, M., y Sola Reche, J. (2016). Diseño y validación de un instrumento para evaluar la competencia digital de los docentes en la Educación Superior española. Píxel-Bit. Revista De Medios Y Educación, (49), 39-6. recuperado a partir de https://recyt.fecyt.es/index.php/pixel/article/view/61713

- Alanís, A. (2001). El saber hacer de la profesión docente. México D.F.: Trillas.

- Alvárez-García, D., Nuñez, J. C., Rodríguez, C., Alvárez L. y Dobarro, A. (2011). Propiedades psicométricas del Cuestionario de Violencia Escolar- Revisado (CUVE-R). Revista de Psicodidáctica, 16 (1), 59-83.

- Ariza Carrasco, C. y Muñoz González, J. M. (2020). Propiedades psicométricas de una escala sobre el aprendizaje del mapa mental aumentado en puzle en Educación Superior. Revista complutense de educación, 31(3), 295-306.

- Boletín Oficial de las Cortes Generales (2020). BOCG, de 25 de noviembre de 2020, página 11.

- Cabero, J., Fernández, B. y Marín, V. (2017). Dispositivos móviles y realidad aumentada en el aprendizaje del alumnado universitario. Revista Iberoamericana de Educación a Distancia, 20(2), 167-185. doi: https://doi.org/10.5944/ ried.20.2.17245

- Canales, R. y Marqués, P. (2007). Factores de buenas prácticas educativas con apoyo de las TIC. Análisis de su presencia en tres centros educativos. Educar, 39; 115-133

- Castañeda, A., del Moral, G., y Suárez, C. (2017). Variables psicológicas comunes en la violencia escolar entre iguales y la violencia filio-parental: un estudio cualitativo. Criminalidad, 59(3), 141-152.

- Castillo, S. y Cabrerizo, J. (2007): Evaluación educativa y promoción escolar. Madrid. Prentice Hall. Pearson.

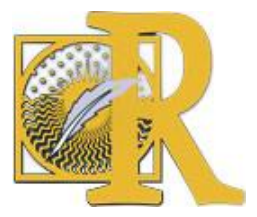

Fecha de recepción: 30-01-2021 Fecha de aceptación: 09-02-2021

Racero-Montes, F. J., Ramos-Corpa, M. J. \& Castillo-García, M. (2021). Validación de un instrumento de práctica docente no presencial en Formación Profesional

International Journal of Educational Research and Innovation (IJERI), 15, 444-459 ISSN: 2386-4303 DOI https://doi.org/10.46661/ijeri.5634 
- Castillo García, M., Ramos Corpas, M. J., y Del Moral Arroyo, G. (2020). Buenas prácticas en la organización, gestión y dirección de los centros educativos. Bordón Revista De Pedagogía, 72(1), 11-30. https://doi.org/10.13042/Bordon.2020.01.63135

- Cava, M.J. y Buelga, S. (2018). Propiedades psicométricas de la Escala de Ciber-Violencia en Parejas Adolescentes (Cib-VPA). Revista Suma Psicológia 25 (2018), 51-61. Doi: http://dx.doi.org/10.14349/sumapsi.2018.v25.n1.6

- Cava, M.J., Povedano, A., Buelga, S., Musitu,G. (2015). Análisis psicométrico de la Escala de Ajuste Escolar Percibido por el Profesor. Psychosocial Intervention, 24 (2), 63-69, https://doi.org/10.1016/..psi.2015.04.001

- Consejería de Educación e Investigación de Madrid (2019). Decreto 63/2019, de 16 de julio, del Consejo de Gobierno, por el que se regula la ordenación y organización de la formación profesional en la Comunidad de Madrid.

- Coll, C. (2007). Las competencias en la educación escolar -algo más que una moda y mucho menos que un remedio. Aula de Innovación Educativa (161), 34-39.

- De la Torre, S. (2000). El profesorado que queremos. En S. De la Torre y O. Barrios (Coords.), Estrategias didácticas innovadoras. Recursos para la formación y el cambio (pp. 95-107). Barcelona: Ediciones Octaedro.

- DESECO (2003). Key competencies for a Successful life and well-functioning society. Final Report. París, Francia: OECD.

- Dorantes - Nova, Judith Araceli, y Hernández - Mosqueda, José Silvano, y Tobón - Tobón, Sergio (2016). Juicio de expertos para la validación de un instrumento de medición del síndrome de Burnout en la docencia. Ra Ximhai, 12(6),327-346. Recuperado de: https://www.redalyc.org/articulo.oa?id=461/46148194023

- Escobar-Pérez, J. y Cuervo-Martínez, A. (2008). Validez de contenido y juicio de expertos: una aproximación a su utilización. Avances en Medición, 6, 27-36.

- Furlong, M. J., Morrison, G. M., Cornell,D. G., \& Skiba, R. (2004).Methodological and measurement issues in school violence research: Moving beyond the social problem era. Journal of School Violence, 3(2-3), 5-12.

- Gamboa, J.P., Moso, M., Albizu, M., Lafuente, A., Mondaca, A., Murciego, A., Navarro, M., y Ugalde, E. (2020). Observatorio de la Formación Profesional en España. Informe 2020. Madrid: Fundación Bankia por la Formación Dual.

- George, D. \& Mallery, P. (2003). SPSS for Windows step by step: A simple guide and reference, Frequencies. 11, 20-52.

- Lara, L., Saracostti, M., Navarro, J.J., de Toro, X., Miranda-Zapata, E., Trigger, J.M., y Fuster, J. (2018). Compromiso escolar: Desarrollo y validación de un instrumento. Revista Mexicana de Psicología, 35(1), 52-62.

- Lei, P.W. (2009). Evaluating estimation methods for ordinal data in structural equation modeling. Quality y Quantity, 43, 495-507. http://doi.org/10.1007/s11135-007-9133-z

- Lizasoain, L. y Angulo, A. (2014). Buenas prácticas de escuelas eficaces del País Vasco. Metodología y primeros resultados. Participación educativa segunda época, 3 (4), extra, 17-27.

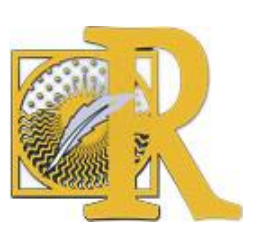

Fecha de recepción: 30-01-2021 Fecha de aceptación: 09-02-2021 
- León-Carrascosa, V., Belando-Montoro, M.R., Sánchez-Serrano, S. (2020). Diseño y validación de un cuestionario para evaluar la metodología Aprendizaje-Servicio. Estudios Sobre Educación, 39, 247-266. DOI: 10.15581/004.39.247-266.

- López-Belmonte, J., Pozo Sánchez, S., Ávila Rodríguez, M., y Montero Cáceres, C. (2020). Proyección pedagógica de la competencia digital docente. El caso de una cooperativa de enseñanza. IJERI: International Journal of Educational Research and Innovation, 14, 167179. https://doi.org/10.46661/ijeri.3844

- Marquès, P. (2000). Cambios en los centros educativos: construyendo la escuela del futuro. http://dewey.uab.es/pmarques/docentes.htm

- MECD (1985). Ley Orgánica 8/1985, de 3 de julio, reguladora del derecho a la Educación. (BOE 4-7-1985)

- MECD (2006). Ley Orgánica 2/2006, de 3 de mayo, de Educación (Texto consolidado, 3009-2020).

- MECD (2013). Ley Orgánica 8/2013, de 10 de diciembre, de mejora de la calidad de la educación (BOE de 9 de diciembre de 2013).

- MECD (2020). Ley Orgánica 3/2020, de 29 de diciembre, por la que se modifica la Ley Orgánica 2/2006, de 3 de mayo, de Educación. (BOE de 30 de diciembre de 2020).

- Muñoz González, J.M., Hidalgo Ariza, M.D., Marín Díaz, V. (2020). Validación de una escala de medida del mapa mental como estrategia de aprendizaje en la formación inicial docente. Estudios Sobre Educación, vol. 38, 79-100. DOI: 10.15581/004.38.79-100.

- Perrenoud, P. (2004). Diez nuevas competencias para enseñar, Barcelona: Graó.

- Ramos Corpas, M. J. y Castillo García, M. (2013). Buenas prácticas en los institutos de educación secundaria. Una práctica orientada a la utilización de la técnica sociométrica para profundizar en el conocimiento del alumnado. Avances en Supervisión Educativa: Revista de la Asociación de Inspectores de Educación de España, 18, 1-10.

- Ramos Corpas, M.J. (2013). Convivencia escolar y buenas prácticas. IC Editorial.

- Ronquillo Triviño, L., Cabrera García, C., y Barberán Cevallos, J. (2019). Competencias profesionales: desafíos en el proceso de formación profesional. Opuntia Brava, 11 (Especial 1), 1-12. Recuperado de: http://200.14.53.83/index.php/opuntiabrava/article/view/653

- Santos-Pastor, M.L.; Cañadas, L.; Martínez-Muñoz, L.F., y García-Rico, L. (2020). Diseño y validación de una escala para evaluar el aprendizaje-servicio universitario en actividad física y deporte. Educación XX1, 23(2), 67-93, doi: 10.5944/educXX1.25422

- Sarramona, J. (1998). Una propuesta para la formación del profesorado. En Fundación Santillana (Ed.), Aprender para el futuro. La educación secundaria, pivote del sistema educativo: documentos de un debate (pp.161-168). Madrid: Fundación Santillana.

- Squires, G. (2004). Localice los problemas de su docencia. Barcelona: CEAC.

- Tejada, J. (2008). Estrategias didácticas para adquirir conocimientos. En Estrategias didácticas en el aula. Buscando la calidad y la innovación (117-130). Madrid: UNED.

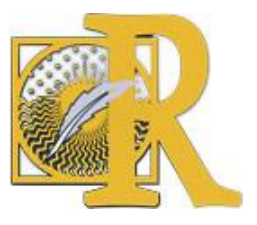

Fecha de recepción: 30-01-2021 Fecha de aceptación: 09-02-2021 